\title{
Impact of Muscle Mass Depletion on Surgical Outcomes in Patients with Primary High-grade Serous Ovarian Cancer Undergoing CRS and HIPEC
}

\author{
Umit Mercan ${ }^{1}$, Basak Gulpinar ${ }^{2}$, Ogun Ersen $^{1}$, Koray Kosmaz $^{3}$, Abdullah Durhan $^{3}$ and Ali Ekrem Unal ${ }^{1}$ \\ ${ }^{1}$ Department of Surgical Oncology, Faculty of Medicine, Ankara University, Ankara, Turkey \\ ${ }^{2}$ Department of Radiology, Faculty of Medicine, Ankara University, Ankara, Turkey \\ ${ }^{3}$ General Surgery Clinic, Ankara Training and Research Hospital, Ankara, Turkey
}

\begin{abstract}
Objective: To investigate the effect of sarcopenia on surgical outcomes in patients with primary high-grade serous ovarian cancer undergoing cytoreductive surgery and hyperthermic intraperitoneal chemotherapy (CRS and HIPEC).

Study Design: Descriptive study.

Place and Duration of Study: Department of Surgical Oncology, Faculty of Medicine, Ankara University Ankara, Turkey, from January 2013 to January 2020.

Methodology: Forty patients, who underwent CRS and HIPEC with the diagnosis of primary high-grade serous ovarian cancer and peritoneal carcinomatosis were included in the study. Preoperative staging CT images were used to determine total psoas Index values by measuring psoas muscle area at the level of L3 vertebra. Patients with total psoas index values below the cutoff levels were considered sarcopenic. Demographic, clinicopathological and perioperative results were compared between sarcopenic and non-sarcopenic patients.

Results: Serious postoperative complications ( $36.4 \%$ vs. $6.9 \%, p=0.039)$ and infective $(54.5 \%$ vs. $17.2 \%, p=0.042)$ and pulmonary $(72.7 \%$ vs. $34.5 \%, p=0.040$ complications were significantly higher in sarcopenic patients. Hospital stay [20(12-25) vs. 12(9-16.5) days, $p=0.017]$ and ICU stay [4(2-6) vs. 2(1-2.5) days, $p=0.013)]$ were found to be longer in sarcopenic patients. In univariate analysis, advanced age (OR: $1.4095 \% \mathrm{Cl}: 1.07-1.84 ; \mathrm{p}=0.021)$ and sarcopenia (OR: $7.71 \% 95 \mathrm{Cl}: 1.17-51.06$; $p=0.039$ ) were significantly associated with serious postoperative complications. The presence of sarcopenia (OR: $0.05095 \%$ $\mathrm{Cl}: 0.004-0.675 ; p=0.024)$ was found to be independent predictor of serious postoperative complications.

Conclusion: Sarcopenia can easily be diagnosed without additional cost or radiation exposure with routine preoperative staging CT images. Identification of sarcopenic ovarian cancer patients in preoperative period may affect patient selection, predictability of possible serious complications, elective operation preparation process with a combination of nutrition and exercise therapy, thus postoperative complication rates may be reduced and short-term results may be improved.
\end{abstract}

Key Words: Cytoreductive surgery, Ovarian cancer, Sarcopenia.

How to cite this article: Mercan U, Gulpinar B, Ersen O, Kosmaz K, Durhan A, Unal AE. Impact of Muscle Mass Depletion on Surgical Outcomes in Patients with Primary High-grade Serous Ovarian Cancer Undergoing CRS and HIPEC. J Coll Physicians Surg Pak 2021; 31(11):1314-1319.

\section{INTRODUCTION}

Ovarian cancer $(\mathrm{OC})$ is the leading cause of cancer-related deaths among all gynecological cancers, and its incidence reaches $2 \%$ in women over the age of 50 years. ${ }^{1}$

Correspondence to: Dr. Umit Mercan, Department of Surgical Oncology, Faculty of Medicine, Ankara University, Ankara, Turkey

E-mail: umit.mercan@yahoo.com.tr

Received: January 18, 2021; Revised: April 18, 2021;

Accepted: October 06, 2021

DOI: https://doi.org/10.29271/jcpsp.2021.11.1314
Despite the development and experience gained in the diagnosis, staging and treatment of ovarian cancer in recent years, high recurrence rates, long-term oncologic results and overall survival rates have not reached satisfactory levels. ${ }^{2}$ The low probability of distant metastasis and the progression of the disease mostly in the form of local invasion and peritoneal spread suggested that successful results obtained in other gastrointestinal cancers with cytoreductive surgery (CRS) and hyperthermic intraperitoneal chemotherapy (HIPEC) can also be achieved in ovarian cancer. In addition to the positive effect of CRS and HIPEC treatment on oncological results, high cellular stress caused by complex surgical procedures; and cytotoxicity and immunosuppression after chemotherapy can cause serious postoperative complications and even mortality. ${ }^{3}$ 
The low performance status of the patients, cancer cachexia and malnutrition also negatively affect postoperative results. Therefore, the preoperative physical condition of the patients is one of the issues that should be considered in the preoperative period. Sarcopenia, which is observed especially in elderly patients and expressed as progressive loss of muscle mass, has also been studied many times in recent years and has been shown to be perhaps the most important objective indicator of physical condition. ${ }^{4}$

Many studies have shown that sarcopenia is an independent prognostic factor on postoperative outcomes in gastrointestinal cancers such as pancreatic, hepatic and biliary malignancies, and urological cancers. ${ }^{5-8}$ In the present study, patients with TPI values below the cut-off levels that were determined by Kim et al., according to age and gender, were considered sarcopenic. ${ }^{9}$

The aim of the study was to determine effect of sarcopenia on surgical outcomes in patients who underwent CRS and HIPEC with the diagnosis of primary high grade serous ovarian cancer and peritoneal carcinomatosis.

\section{METHODOLOGY}

This is a descriptive study conducted at the Department of Surgical Oncology, Faculty of Medicine, Ankara University, Ankara, Turkey from January 2013 to January 2020. Forty patients who underwent CRS and HIPEC, with the diagnosis of primary high-grade serous ovarian carcinoma and peritoneal carcinomatosis and whose psoas muscle mass measurements could be obtained, were included in the study. Patients' demographic data, laboratory results, pathology results and all data related to postoperative results were retrospectively reviewed by surgical oncologists through the hospital database. Patients whose data could not be accessed, who had recurrent disease, low grade tumors, and who could not undergo CRS and HIPEC considering that CC-0/1 cytoreduction could not be performed by intraoperative exploration, were excluded from the study. Clinical and demographic data and perioperative outcomes were compared between sarcopenic and non-sarcopenic groups. Postoperative complications were determined on the basis of the modified clavien dindo classification (CD), and grade three or higher complications were accepted as serious complications. ${ }^{10}$

Interval cytoreduction was planned for patients who were diagnosed with FIGO stage IIIC primary high-grade serous ovarian cancer and achieved stable disease or remission after three cycles of neoadjuvant chemotherapy.The anatomical extension of the $O C$ in the peritoneal cavity was evaluated by the $\mathrm{PC}$ index $(\mathrm{PCl}){ }^{11}$ CRS desicion was made at the time of surgery and it was applied in cases where CC-0 (no visible tumor) or CC-1(tumor nodules persisting after cytoredction are less than $2.5 \mathrm{~mm}$ in diameter) cytoreduction waspredicted to be performed. ${ }^{12}$ HIPEC wassimultaneously performed in each patient with CC- 0 and 1 cytoreduction.

In preoperative staging abdominal computerised tomography (CT) scans, the right and left psoas muscle areas at the level of the L3 vertebra were measured three consecutive times in each patient by two independent radiologists. The average of the values obtained was determined as the psoas muscle area. The measurement of the psoas muscle at the third lumbar vertebra level and the calculation of the total psoas Index (TPI) with patients' height have been described in previous studies. ${ }^{13} \mathrm{TPI}$ is calculated by dividing the total area of the right and left psoas muscles by the square of the individual's height in meters [(right psoas muscle area + left psoas muscle area) / height $(\mathrm{m})^{2}$ ]. Patients with TPI values below the cut-off levels determined according to age groups and gender by Kim et al. were considered sarcopenic $\left(4 \mathrm{~cm}^{2} / \mathrm{m}^{2}\right.$ for $20-39$ years age; $2.88 \mathrm{~cm}^{2} / \mathrm{m}^{2}$ for $40-49$ years age; $2.43 \mathrm{~cm}^{2} / \mathrm{m}^{2}$ for $50-59$ years age; $2.20 \mathrm{~cm}^{2} / \mathrm{m}^{2}$ for $60-69$ years age and $1.48 \mathrm{~cm}^{2} / \mathrm{m}^{2}$ for $70-89$ years age). The patients were divided into two groups as sarcopenic and non-sarcopenic.

Numerical data were expressed as mean \pm standard deviation, median (IQR: 25th percentile-75th percentile) or percentages. The normal distribution of the data was determined by histogram graphics and the Shapiro-WilkTest. In comparison of clinical and demographic data and perioperative results between sarcopenic and non-sarcopenic patients, Chi-square test or Fisher Exact test were used for categorical variables. Studentt-testor Man-WhitneyU-test was carried outfornumerical variables. Binary logistic regression analysis was used to determine the factors affecting serious postoperative complications. All p-values less than 0.05 were considered statistically significant. These analyses were performed using IBM ${ }^{\circledR}$ SPSS statistic version 23.0.

\section{RESULTS}

According to the cut-off levels determined, while there were no sarcopenic patients in the 20-39 years age group, a total of 11 (27.5\%) patients were diagnosed as sarcopenic in other age groups (1, 2, 4 and 4 patients in age groups of 40-49, 50-59, 60-69 and 70-89 years, respectively). The mean TPI value was $2.06 \pm 0.48$ in the sarcopenic group, and $3.73 \pm 0.82$ in the nonsarcopenic group $(p<0.001)$.

The relationship between demographic and clinical variables with sarcopenia is summarised in Table I. The median age of the patients included in the study was 64.0 (54.25-69.0) years. Although it was found that patients in the sarcopenic group had lower mean body mass index (BMI) values compared to the other group and there were more patients in the ASAlll group, the difference was not statistically significant.

The relationship between perioperative results and sarcopenia is summarised in Table II. While there was no difference between the groups in terms of $\mathrm{PCl}, \mathrm{CC}$ score, operation time, intraoperative blood loss and in-hospital mortality, it was observed that serious complications of modified CD grade 3 and above were significantly more common in the sarcopenic group (36.4\% vs. $6.9 \% ; p=0.039$ ). 


\begin{tabular}{|c|c|c|c|c|}
\hline Variables & $\begin{array}{c}\text { Total } \\
(n=40)\end{array}$ & $\begin{array}{c}\text { Sarcopenic } \\
(n=11)\end{array}$ & $\begin{array}{c}\text { Non-sarcopenic } \\
(n=29)\end{array}$ & pvalue \\
\hline Age & $64.0(54.25-69.0)$ & $67.0(54.0-73.0)$ & $62.0(54.0-69.0)$ & 0.339 \\
\hline BMI $\left(\mathrm{kg} / \mathrm{m}^{2}\right)$ & $28.95(24.15-32.38)$ & $30.08(22.04-32.37)$ & $28.19(24.17-32.39)$ & 0.868 \\
\hline $\begin{array}{l}\text { ASA score } n(\%) \\
\text { II } \\
\text { III }\end{array}$ & $\begin{array}{l}14(35) \\
14(35) \\
12(30)\end{array}$ & $\begin{array}{l}2(18.2) \\
4(36.4) \\
5(45.4)\end{array}$ & $\begin{array}{c}12(41.4) \\
10(34.5) \\
7(24.1)\end{array}$ & 0.372 \\
\hline $\begin{array}{l}\text { Comorbid diseases } n(\%) \\
\text { HT } \\
\text { DM } \\
\text { Cardiac } \\
\text { Pulmonary }\end{array}$ & $\begin{array}{c}22(55) \\
11(27.5) \\
9(22.5) \\
8(20)\end{array}$ & $\begin{array}{l}7(63.6) \\
5(45.4) \\
4(36.4) \\
3(27.3)\end{array}$ & $\begin{array}{l}15(51.7) \\
6(20.7) \\
5(17.2) \\
5(17.2)\end{array}$ & $\begin{array}{l}0.723 \\
0.137 \\
0.227 \\
0.660\end{array}$ \\
\hline CA-125 (IU/ml) & $254.5(169-340.75)$ & $280(183-345)$ & $238(168-339.5)$ & 0.515 \\
\hline Albumin (g/dl) & $4.11(3.78-4.37)$ & $3.93(3.61-4.32)$ & $4.11(3.84-4.42)$ & 0.596 \\
\hline $\begin{array}{l}\text { Ascites (\%) } \\
\text { None } \\
\text { Minimal/medium } \\
\text { Massive }\end{array}$ & $\begin{array}{l}13(32.5) \\
20(50) \\
7(17.5)\end{array}$ & $\begin{array}{l}3(27.3) \\
6(54.5) \\
2(18.2)\end{array}$ & $\begin{array}{c}10(34.5) \\
14(48.3) \\
5(17.2)\end{array}$ & 0.906 \\
\hline $\operatorname{TPI}\left(\mathrm{cm}^{2} / \mathrm{m}^{2}\right)$ & $3.27 \pm 1.05$ & $2.06 \pm 0.48$ & $3.73 \pm 0.82$ & $<0.001$ \\
\hline
\end{tabular}

Table II: The relationship between perioperative results and sarcopenia.

\begin{tabular}{|c|c|c|c|c|}
\hline Variables & $\begin{array}{c}\text { Total } \\
(n=40)\end{array}$ & $\begin{array}{c}\text { Sarcopenic } \\
(n=11)\end{array}$ & $\begin{array}{c}\text { Non-sarcopenic } \\
(n=29)\end{array}$ & p-value \\
\hline $\begin{array}{l}\mathrm{PCl} * \mathrm{n}(\%) \\
\mathrm{PCl} \leq 5 \\
5 \\
\mathrm{PCl} \geq 10\end{array}$ & $\begin{array}{c}2(5) \\
11(27.5) \\
27(67.5)\end{array}$ & $\begin{array}{c}1(9.1) \\
2(18.2) \\
8(72.7)\end{array}$ & $\begin{array}{c}1(3.4) \\
9(31) \\
19(65.5)\end{array}$ & 0.477 \\
\hline $\begin{array}{l}\text { CC score** n(\%) } \\
\text { CC-0 } \\
\text { CC-1 }\end{array}$ & $\begin{array}{l}29(72.5) \\
11(27.5)\end{array}$ & $\begin{array}{l}9(81.8) \\
2(18.2)\end{array}$ & $\begin{array}{l}20(69) \\
9(31)\end{array}$ & 0.694 \\
\hline Operation time (min) & $205.65 \pm 25.04$ & $206.82 \pm 23.37$ & $205.21 \pm 26.04$ & 0.859 \\
\hline Intraoperativebloodloss (>1000 cc) n(\%) & $6(15)$ & $2(18.2)$ & $4(13.8)$ & $>0.999$ \\
\hline$C D \geq$ grade 3 complications*** $n(\%)$ & $6(15)$ & $4(36.4)$ & $2(6.9)$ & 0.039 \\
\hline $\begin{array}{l}\text { Postoperative complications } \mathrm{n}(\%) \\
\text { Cardiac complications } \\
\text { Pulmonary complications } \\
\text { Infective complications } \\
\text { Wound complications } \\
\text { Acute renal failure }\end{array}$ & $\begin{array}{c}6(15) \\
18(45) \\
11(27.5) \\
6(15) \\
5(12.5)\end{array}$ & $\begin{array}{l}2(18.2) \\
8(72.7) \\
6(54.5) \\
3(27.3) \\
2(18.2)\end{array}$ & $\begin{array}{l}4(13.8) \\
10(34.5) \\
5(17.2) \\
3(10.3) \\
3(10.3)\end{array}$ & $\begin{array}{c}>0.999 \\
0.040 \\
0.042 \\
0.319 \\
0.603\end{array}$ \\
\hline Hospital stay (day) & $13.50(10.25-20.75)$ & $20(12-25)$ & $12(9-16.5)$ & 0.017 \\
\hline ICU stay (day) & $2(1-4)$ & $4(2-6)$ & $2(1-2.5)$ & 0.013 \\
\hline In-hospital mortality n(\%) & $3(7.5)$ & $1(9.1)$ & $2(6.9)$ & $>0.999$ \\
\hline \multicolumn{5}{|c|}{$\begin{array}{l}\text { Numerical data are given as mean } \pm \text { standard deviation, median(IQR) or percentages PCI: Peritoneal CarcinomatozisIndex, CC: Completeness of } \\
\text { Cytoreduction, CD: Clavien Dindo, ICU: Intensive Care Unit. *The burden of the peritoneal disease was determined according to the PCI defined by } \\
\text { Sugarbaker et al. }{ }^{11}{ }^{* *} \text { The extent of cytoreduction was determined according to CC score defined by Harmon et al. }{ }^{12}{ }^{* * *} \text { Postoperative complications were } \\
\text { determined according to the modified Clavien Dindo classification. }{ }^{10}\end{array}$} \\
\hline
\end{tabular}

When postoperative complications were categorised, it was found that pulmonary and infective complications were significantly more common in the sarcopenic group $(p=$ 0.040 , and $p=0.042$, respectively). It was observed that sarcopenia significantly prolonged hospital stay and intensive care unit stay ( $p=0.017$, and $p=0.013$, respectively).

As a result of univariate analysis, age $>65$ years (OR: 1.40 95\% Cl: 1.07-1.84; $p=0.021$ ), and sarcopenia (OR:7.71 95 $\mathrm{Cl}: 1.17-51.06 ; p=0.039)$ were found to be effective factors with regard to serious postoperative complications. In multivariate analysis, sarcopenia (OR: 0.05095\% Cl: 0.004-0.675; $p=0.024$ ) was found to be independent predictor of serious postoperative complications.

\section{DISCUSSION}

Primary treatment of ovarian cancers with locally advanced and peritoenal disease is neodjuvant chemotherapy followed by interval cytoreduction surgery. ${ }^{14}$ Although improved oncological results could be achieved in many studies by combining HIPEC with cytoreduction, the cellular stress and cytotoxic effect of complex surgeries and chemotherapy may cause serious postoperative complications and 
morbidity. ${ }^{15}$ In addition, the low performance status of the patients also has a negative effect on survival and short-term outcomes.

Cancer cachexia, which is characterised by involuntary weight loss and severe muscle mass loss due to the effect of tumoral factors, is observed in patients with advanced stage ovarian cancer and this is one of the most important factors that reduces the performance status of patients. ${ }^{16}$ Primary mechanisms that cause cancer-related sarcopenia include the usage of endogenous amino acids by the liver to produce antitumor proteins by consuming skeletal muscles inefficiently, excessive release of pro-inflammatory mediators targeting muscle tissue, impaired oral food intake, and catabolic state resulting from abnormal metabolic activity. ${ }^{17,18}$ The severity of the catabolic process and the resulting muscle depletion are correlated with the burden of the disease. Although it was not statistically significant in present study, it was observed that $\mathrm{PCl}$ was above 10 in a majority of sarcopenic patients. In addition, many patients diagnosed with ovarian cancer presented with widespread disease and the resulting massive ascites, which may often mask muscle depletion and weight loss. The lack of difference in terms of BMI between the sarcopenic group and the non-sarcopenic group in present study can be explained in this way.

Sarcopenia is defined as a syndrome characterised by progressive loss of muscle mass resulting in physical disability and in its diagnosis, radiological methods based on muscle mass or area measurements such as X-ray absorptiometry, magnetic resonance imaging, CT and bioelectrical impedance analysis are used in addition to muscle strength and physical performance measurements. ${ }^{4}$ Although CT is defined as the gold standard method in measuring muscle mass or quality, it has disadvantages such as high cost, limited trained personnel and radiation exposure. ${ }^{19}$ However, $\mathrm{CT}$ is routinely ordered for preoperative staging in ovarian cancer patients, and therefore, its use for sarcopenia evaluation will not increase the cost or cause extra $\mathrm{X}$-ray exposure. For these reasons, muscle mass measurements were performed using preoperative staging CT images in the present study.

There are many methods for measuring muscle mass including different anatomical regions described in the literature. ${ }^{20}$ In the present study, psoas muscle area measurements at the L3 vertebra level were used. Then, a TPI value was calculated for each individual with these measurements. Determination of sarcopenia according to TPI value has been described earlier in previous studies. ${ }^{13}$ Although this method can be easily applied in clinical practice in many cancer patients, the main problem in its use for the diagnosis of sarcopenia is the determination of cutoff level for the accurate diagnosis. Although there is no consensus in the literature on this subject, there are studies in which different cut- off levels have been determined for different age groups and races. $^{20 .}$ It is natural that there is a difference between the muscle mass of elderly and younger patients due to physiological muscle mass depletion in the elderly. Since the proportion of fatty mass contained in muscle tissue is different from each other in both genders, the average muscle mass in men is higher than in women. For these reasons, the TPI cut-off levels determined according to different age groups; and gender defined by Kim et al. were used in the diagnosis of sarcopenia in present study. ${ }^{9}$

Many patient and surgery-related factors play a role in the development of postoperative complications. In the present study, serious postoperative complications requiring surgical intervention or life-threatening were observed more frequently in sarcopenic patients. Hospital and ICU stay were found to be significantly higher in sarcopenic patients due to complications developing in the early postoperative period and the delay in the mobilization of the patient due to poor performance and muscle weakness. Increased presence of pulmonary complications such as pneumomia and atelectasis in sarcopenic patients was due to the impairment of lung expansion caused by loss of respiratory muscles and pain caused by extensive surgical resections and surgical incisions performed. Additionally, traumatic effect resulting from wide diaphragmatic peritoneal dissection and pleural effusion due to diffusion of chemotherapeutics into the thoracic cavity aggravates respiratory distress.

Similarly, in a systematic review, conducted by Pamoukdjian et al., sarcopenia in various cancer types was found to be associated with postoperative pulmonary complications such as atelectasis, pulmonary embolism and pneumonia. ${ }^{21}$ Therefore, caution should be exercised in terms of respiratory failure, especially in elderly and sarcopenic patients with chronic lung disease. In addition, the inflammatory response and immunosuppression resulting from the cellular stress after cytoreductive surgery and the local cytotoxic effect caused by the HIPEC administiration are more severe in sarcopenic patients who are in catabolic process and have impaired nutritional status. ${ }^{22,23}$ Therefore, it is considered that infective complications are more frequent in sarcopenic patients. Contrary to the present results, in a study conducted by Rutten et al., a significant relationship was not found between postoperative complications and sarcopenia in patients who underwent CRS for ovarian cancer. ${ }^{24}$

The main limitations of the study are possible selection bias due to being of a retrospective study and relatively low sample size. In addition, the fact that there are different methods used in the diagnosis of sarcopenia in the literature and the cut-off values determined for each method are different from each other affect the generalizability of the study results for different populations. Moreover, sarcopenia should be defined not only with muscle mass but also with muscle strength and physical performance but the authors 
were not able to gather data on other parameters due to the study's retrospective design. Additional prospective studies including questionnaires for sarcopenia-associated symptoms and including measurements of muscle strength and detailed physical performance are needed to better define the sarcopenia and to better understand its effect on postoperative outcomes.

\section{CONCLUSION}

Sarcopenia was found to be an independent predictor of postoperative complications in patients undergoing CRS and HIPEC for ovarian cancer. Sarcopenia can be easily diagnosed without additional cost or radiation exposure with routine preoperative staging CT images. Identification of sarcopenic patients in preoperative period may affect patient selection, predictability of possible serious complications, elective operation preparation process with a combination of nutrition and exercise therapy, thus postoperative complication rates may be reduced and short-term results may be improved.

\section{ETHICAL APPROVAL:}

Faculty of Medicine Ethic Committee, Ankara University approved the study in January 2020. Approval date and No. 16.01.2020, 11-38-20.

\section{PATIENTS' CONSENT:}

The patients' consents were waived since the present study was retrospective.

\section{CONFLICT OF INTEREST:}

The authors have no conflict of interest to declare.

\section{AUTHORS' CONTRIBUTION:}

UM: Data collection, statistical analysis and writing the manuscript.

BG: CT measurements.

OE: Data collection and literature review.

KK: Data collection and study design.

AD: Literature review and writing.

AEU: Critical analysis and managing the manuscript.

\section{REFERENCES}

1. Coleman RL, Monk BJ, Sood AK, Herzog TJ. Latest research and treatment of advanced-stage epithelial ovarian cancer. Nat Rev Clin Oncol 2013; 10(4):211-24. doi: 10.1038/nrclinonc.2013.5.

2. Ubachs J, Ziemons J, Minis-Rutten IJ, Kruitwagen RF, Kleijnen J, Lambrechts S, et al. Sarcopenia and ovarian cancer survival: A systematic review and meta-analysis. J Cachexia Sarcopenia Muscle 2019; 10(6):1165-74. doi: 10.1002/jcsm.12468.

3. Khatri VP. Cytoreductive surgery and hyperthermic Intraperitoneal chemotherapy for colorectal cancer: A panacea or just an obstacle course for the patient? J Clin Oncol 2010; 28(1):5-7. doi: 10.1200/JCO.2009.24.8500.

4. Cruz-Jentoft AJ, Baeyens JP, Bauer JM, Boirie Y, Cederholm T,
Landi F, et al. Sarcopenia: European consensuson definitionand diagnosis: Report of the european working group on sarcopeniainolder people. Age Ageing 2010; 39(4):412-23. doi: 10.1093/ageing/afq034.

5. Choi Y, Oh DY, Kim TY, Lee KH, Han SW, Im SA, et al. Skeletal muscle depletion predicts the prognosis of patients with advanced pancreatic cancer undergoing palliative chemotherapy, independent of body mass index. PLoS One 2015; 10(10):e0139749. doi: 10.1371/journal.pone.0139 749.

6. Levolger S, Van Vugt JL, De Bruin RW, IJzermans JN. Systematic review of sarcopenia in patients operated on for gastrointestinal and hepatopancreatobiliary malignancies. BrJ Surg 2015; 102(12):1448-58. doi: 10.1002/bjs.9893.

7. Shachar SS, Williams GR, Muss HB, Nishijima TF. Prognostic value of sarcopenia in adults with solid tumours: A meta-analysis and systematic review. Eur J Cancer 2016; 57:58-67. doi: 10.1016/j.ejca.2015.12.030.

8. Fukushima H, Nakanishi Y, Kataoka M, Tobisu KI, Koga F. Prognostic significance of sarcopenia in upper tract urothelial carcinoma patients treated with radical nephroureterectomy. Cancer Med 2016; 5(9):2213-2220. doi: 10.1002/cam4.795.

9. Kim JS, Kim WY, Park HK, Kim MC, Jung W, Ko BS. Simple age specific cutoff value for sarcopenia evaluated by computed tomography. Ann Nutr Metab 2017; 71(3-4): 157-163. doi: 10.1159/000480407.

10. Dindo D, Demartines N, Clavien PA. Classification of surgical complications: A new proposal with evaluation in a cohort of 6336 patients and results of a survey. Ann Surg 2004; 240 (2):205-13. doi: 10.1097/01.sla.0000133083.54934.ae.

11. Sugarbaker PH. Peritonectomy procedures. Ann Surg 1995; 221:29-42.

12. Harmon RL, Sugarbaker PH. Prognostic indicators in peritoneal carcinomatosis from gastrointestinal cancer. Int Semin Surg Oncol 2005; 2(1):3. doi: 10.1186/14777800-2-3.

13. Peng P, Hyder O, Firoozmand A, Kneuertz P, Schulick RD, Huang $D$, et al. Impact of sarcopenia on outcomes following resection of pancreatic adenocarcinoma. J Gastrointestinal Surg 2012; 16(8):1478-86. doi: 10.1007/s11605-0121923-5.

14. Vergote I, Tropé CG, Amant F, Kristensen GB, Ehlen T, Johnson $\mathrm{N}$, et al. Neoadjuvant chemotherapy or primary surgery in stage IIIC or IV ovarian cancer. New England J Med 2010; 363(10):943-53. doi: 10.1056/NEJMoa0908806.

15. Chua TC, Robertson G, Liauw W, Farrell R, Yan TD, Morris $\mathrm{DL}$, et al. Intraoperative hyperthermic intraperitoneal chemotherapy after cytoreductive surgery in ovarian cancer peritoneal carcinomatosis: Systematic review of current results. J Cancer Res Clin Oncol 2009; 135(12):1637-45. doi: 10.1007/s00432-009-0667-4.

16. Tisdale MJ. Cachexia in cancer patients. Nature Reviews Cancer 2012; 2(11):862-71.

17. Fearon K, Strasser F, Anker SD, Bosaeus I, Bruera E, Fainsinger RL, et al. Definition and classification of cancer cachexia: An international consensus. The Lancet Oncol 2011; 12(5): 489-495. doi: 10.1016/S1470-2045(10) 
70218-7.

18. Zhang G, Li X, Sui C, Zhao H, Zhao J, Hou Y, et al. Incidence and risk factor analysis for sarcopenia in patients with cancer. Oncology letters 2016; 11(2):1230-4. doi: 10.3892/ol.2015.4019.

19. Boutin RD, Yao L, Canter RJ, Canter RJ, Lenchik L. Sarcopenia: Current concepts and imaging implications. AJR AmJ Roentgenol 2015; 205(3):W255-W66. doi: 10.2214/AJR.15.14635.

20. Walowski CO, Braun W, Maisch MJ, Jensen B, Peine S, Norman K, et al. Reference values for skeletal muscle mass-current concepts and methodological consider-ations. Nutrients 2020; 12(3):755. doi: 10.3390/nu12030755.

21. Pamoukdjian F, Bouillet T, Lévy V, Soussan M, Zelek L, Paillaud $\mathrm{E}$. Prevalence and predictive value of pre-therapeutic sarcopenia in cancer patients: A systematic review. Clinical Nutrition 2018; 37(4):1101-13. doi: 10.1016/j.clnu.2017. 07.010.
22. Chemama S, Bayar MA, Lanoy E, Ammari S, Stoclin A, Goéré $D$, et al. Sarcopenia is associated with chemotherapy toxicity in patients undergoing cytoreductive surgery with hyperthermic intraperitoneal chemotherapy for peritoneal carcinomatosis from colorectal cancer. Annals Surg Oncol 2016; 23(12):3891-8. doi: 10.1245/s10434-016-5360-7.

23. Leijte GP, Custers H, Gerretsen J, Heijne A, Roth J, Vogl T, et al. Increased plasma levels of danger-associated molecular patterns are associated with immune suppression and postoperative infections in patients undergoing cytoreductive surgery and hyperthermic intraperitoneal chemotherapy. Frontiers in immunol 2018; 9:663. doi: 10.3389/fimmu.2018.00663.

24. Rutten IJ, Ubachs J, Kruitwagen RF, van Dijk DP, Beets-Tan $R G$, Massuger LF, et al. The influence of sarcopenia on survival and surgical complications in ovarian cancer patients undergoing primary debulking surgery. Eur J Surg Oncol 2017; 43:717-24. doi: 10.1016/j.ejso.2016.12.016. 